\title{
GENERALIZED AERODYNAMIC FORCES ON A FLEXIBLE PLATE UNDERGOING TRANSIENT MOTION*
}

BY

\author{
EARL H. DOWELL
}

Princeton University

\begin{abstract}
The aerodynamic forces on a flexible plate embedded in a rigid half-space, undergoing arbitrary temporal and spatial motion are determined.

I. Introduction. There are two rather closely related problems involving the motion of a flexible plate in a fluid flow which are of current engineering interest. These are the stability of the fluid-plate system (plate or panel flutter) and the response of the system to "external" forces, e.g., response to pressure fluctuations in a turbulent boundary layer. In either case the determination of the aerodynamic forces due to the plate motion are of interest (though for the latter problem current practice would appear to be to ignore these forces. This seems to be done in order to simplify the calculation rather than on the basis of any assurance that such a neglect is justified). Heretofore only the special case of sinusoidal or simple harmonic motion has been treated [1]. In the present work the more general case of arbitrary time (as well as spatial) dependence of the plate motion is considered.
\end{abstract}

\section{Nomenclature}

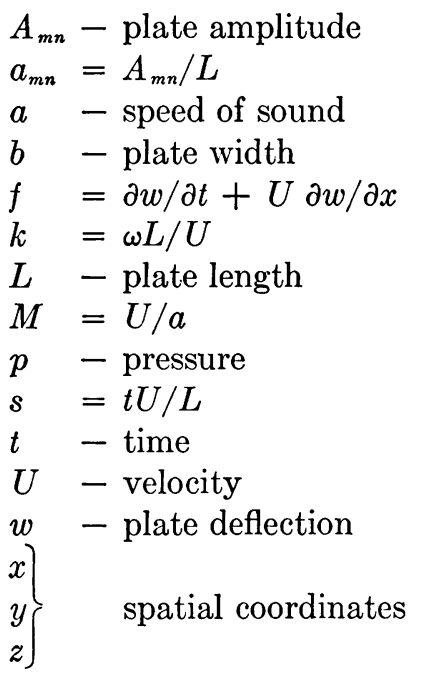

$$
\begin{array}{ll}
\begin{array}{l}
\alpha \\
\gamma
\end{array} & \text { Fourier Transform variables } \\
\xi & =x / L \\
\eta & =y / b \\
\rho & - \text { density } \\
\sigma & =\tau U / L \\
\tau & - \text { dummy time variable } \\
\phi \quad & \text { - velocity potential } \\
\omega & - \text { frequency } \\
\text { Superscripts } \\
, \quad \equiv \partial / \partial \xi \\
\text { D } \equiv \partial / \partial s \\
\text { Subscripts } \\
R \quad \text { - real part } \\
I \quad-\text { imaginary part }
\end{array}
$$

II. Formulation and solution. We consider a flexible, rectangular plate embedded in a rigid halfspace (see Fig. 1). Over the surface of the plate there is a flow of an inviscid, irrotational fluid. (The flow is taken parallel to the $x$ axis; the subsequent analysis

*Received March 22, 1966. 


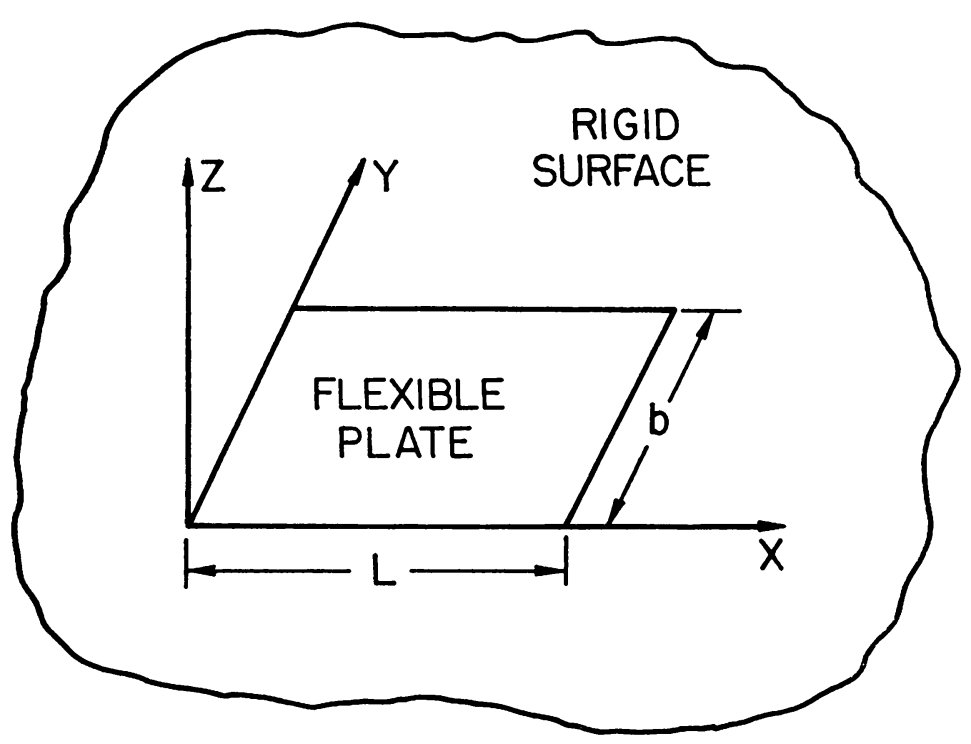

Fig. 1. Problem Geometry

may be generalized to the case of a flow at an arbitrary angle to the $x$ axis in a straightforward manner.) The flexible plate deforms in an arbitrary way with respect to space and time; the object is to determine the fluid (aerodynamic) forces acting on the plate.

Within the framework of linear theory the boundary value-initial value problem may be stated as follows: We seek a solution to the partial differential equation for the velocity potential, $\phi$,

$$
\nabla^{2} \phi-\frac{1}{a^{2}}[\partial / \partial t+U \partial / \partial x]^{2} \phi=0 .
$$

Subject to the boundary condition

$$
\begin{aligned}
\left.\frac{\partial \phi}{\partial z}\right|_{z-0} & =\partial w / \partial t+U \partial w / \partial x \equiv f(x, y, z, t) & & \text { on plate } \\
& =0 & & \text { off plate }
\end{aligned}
$$

and also an appropriate boundary condition as $z \rightarrow \infty$.

This problem will be treated by the transform calculus employing a Laplace Transform with respect to time and a double Fourier Transform with respect to the spatial variables, $x$ and $y$. Defining

$$
\Phi(z, y, x ; p) \equiv \int_{0}^{\infty} e^{-p t} \phi(x, y, z, t) d t
$$

and

$$
\Phi^{*}(z ; \gamma, \alpha, p) \equiv \int_{-\infty}^{\infty} \int_{-\infty}^{\infty} \exp [-i(\gamma y+\alpha x)] \Phi(z, y, x ; p) d y d x
$$

Eqs. (1) and (2) become (assuming that $\phi=\partial \phi / \partial t=0$ at $t=0^{-}$)

$$
d^{2} \Phi^{*} / d z^{2}=\mu^{2} \Phi^{*}
$$


where

$$
\mu \equiv\left[p^{2} / a^{2}+2 M p i \alpha / a-\alpha^{2}\left(M^{2}-1\right)+\gamma^{2}\right]^{1 / 2}
$$

and

$$
d \Phi^{*} / d z=F^{*}
$$

where

$$
F^{*} \equiv \int_{0}^{\infty} \int_{-\infty}^{\infty} \int_{-\infty}^{\infty} e^{-p t} \exp [-i(\gamma y+\alpha x)] f d y d x d t
$$

Solving Eq. (3) subject to (4) gives

$$
\left.\Phi^{*}\right|_{z=0}=-F^{*} / \mu .
$$

(The square root of $\mu$ is to be chosen such that the condition of finiteness or radiation is satisfied at infinity. We will not need to do this explicitly.) Using well-known inversion formulae [2] we may invert into the time domain.

$\phi^{*}(\gamma, \alpha, z=0, t)$

$$
=-a \int_{0}^{t} f^{*}(\gamma, \alpha, \tau) \exp [-i M a \alpha(t-\tau)] \cdot J_{0}\left[\left(\alpha^{2}+\gamma^{2}\right)^{1 / 2} a(t-\tau)\right] d \tau .
$$

Formally the inversion may now be made into the spatial domain. However, instead we proceed with the calculation of the Fourier Transform of the fluid forces. The fluid pressure is related to the velocity potential by the Bernoulli formula,

$$
p=-\rho[\partial \phi / \partial t+U \partial \phi / \partial x] .
$$

From equations (5) and (6) one may compute the Fourier Transform of the pressure as

$$
\begin{aligned}
p_{z-0}^{*}=\rho a\left\{f^{*}(t)-a\left(\alpha^{2}+\gamma^{2}\right)^{1 / 2} \int_{0}^{t} f^{*}(\tau)\right. & \\
& \left.\quad \exp [-i a M \alpha(t-\tau)] J_{1}\left[a\left(\alpha^{2}+\gamma^{2}\right)^{1 / 2}(t-\tau)\right] d \tau\right\} .
\end{aligned}
$$

From this we see the well-known result that at $t=0^{+}$, the pressure is that given by "piston theory." Again a formal inversion into the spatial domain may be made, if desired.

For the usual applications [1] the forces of interest are the "generalized aerodynamic forces" rather than the pressure itself. If

$$
w=\sum_{m} \sum_{n} A_{m n}(t) \psi_{m}(x) X_{n}(y)
$$

then the (nondimensional) generalized force, $Q_{m n p a}$, is defined as

$$
Q_{m n p e} \equiv \frac{\int_{0}^{L} \int_{0}^{b} p_{m n} \psi_{p}(x) X_{q}(y) d x d y}{\rho U^{2} L b}
$$

where $p_{m n}$ is the pressure due to

$$
w=A_{m n}(t) \psi_{m}(x) X_{n}(y) .
$$


A considerable economy of effort may be achieved by performing the integrals over $x$ and $y$ in equation (9) before inverting the Fourier Transform. Having done this, $Q_{m n p a}$ may be written as

$$
\begin{aligned}
Q_{m n p q}(s)= & a_{m n}(s) S_{m n p q}+\dot{a}_{m n}(s) D_{m n p q} \\
& +\int_{0}^{s} a_{m n}(\sigma) H_{m n p q}(s-\sigma) d \sigma \\
& +\int_{0}^{s} \dot{a}_{m n}(\sigma) I_{m n p q}(s-\sigma) d \sigma
\end{aligned}
$$

where

$$
\begin{aligned}
& S_{m n p q} \equiv \frac{1}{M} \int_{0}^{1} \psi_{m}^{\prime}(\xi) \psi_{p}(\xi) d \xi \int_{0}^{1} X_{n}(\eta) X_{q}(\eta) d \eta \\
& D_{m n p q} \equiv \frac{1}{M} \int_{0}^{1} \psi_{m}(\xi) \psi_{p}(\xi) d \xi \int_{0}^{1} X_{n}(\eta) X_{p}(\eta) d \eta \\
& H_{m n p q}(s) \equiv-\frac{1}{4 \pi^{2}} \frac{1}{M^{2}} \int_{-\infty}^{\infty} \int_{-\infty}^{\infty} G_{m n p q} i \alpha^{*}\left(\alpha^{*^{2}}+\gamma^{*^{2}}(L / b)^{2}\right)^{1 / 2} \\
& \cdot \exp \left[-i \alpha^{*} s\right] J_{1}\left[\left(\alpha^{*^{2}}+\gamma^{*^{2}}(L / b)^{2}\right)^{1 / 2} s / M\right] d \alpha^{*} d \gamma^{*}, \\
& I_{m n p q}(s) \equiv-\frac{1}{4 \pi^{2}} \frac{1}{M^{2}} \int_{-\infty}^{\infty} \int_{-\infty}^{\infty} G_{m n p q}\left(\alpha^{*^{2}}+\gamma^{\left.*^{2}(L / b)^{2}\right)^{1 / 2}}\right. \\
& \cdot \exp \left[-i \alpha^{*} s\right] J_{1}\left[\left(\alpha^{*^{2}}+\gamma^{*^{2}}(L / b)^{2}\right)^{1 / 2} s / M\right] d \alpha^{*} d \gamma^{*}, \\
& G_{m n p q} \equiv \int_{0}^{1} \psi_{m}(\xi) \exp \left[-i \alpha^{*} \xi\right] d \xi \int_{0}^{1} \psi_{p}(\xi) \exp \left[i \alpha^{*} \xi\right] d \xi \\
& \cdot \int_{0}^{1} X_{n}(\eta) \exp \left[-i \gamma^{*} \eta\right] d \eta \int_{0}^{1} X_{q}(\eta) \exp \left[i \gamma^{*} \eta\right] d \eta, \\
& \alpha^{*} \equiv \alpha L, \quad \gamma^{*} \equiv \gamma b
\end{aligned}
$$

$s$ is a nondimensional time; see Nomenclature for other definitions. In the derivation it has been assumed that $\psi_{m}(0)=\psi_{m}(1)=X_{n}(0)=X_{n}(1)=0$.

By defining

$$
\begin{aligned}
& g_{m p}\left(\alpha^{*}\right) \equiv \int_{0}^{1} \psi_{m} \exp \left[-i \alpha^{*} \xi\right] d \xi \int_{0}^{1} \psi_{p} \exp \left[i \alpha^{*} \xi\right] d \xi \\
& g_{n a}\left(\gamma^{*}\right) \equiv \int_{0}^{1} X_{n} \exp \left[-i \gamma^{*} \eta\right] d \eta \int_{0}^{1} X_{q} \exp \left[i \gamma^{*} \eta\right] d \eta
\end{aligned}
$$

and noting that the real and imaginary parts of $g_{n p}, g_{n q}$ are even and odd functions of their arguments, respectively; the expressions for $H_{\text {mnpq }}(s)$ and $I_{m n p q}(s)$ may be simplified to

$H_{m n p Q}=-\frac{1}{\pi^{2}} \frac{1}{M^{2}} \int_{0}^{\infty}\left(g_{n a}\right)_{R}\left\{\int_{0}^{\infty} \alpha^{*}\left[\left(g_{m p}\right)_{R} \sin \alpha^{*} s-\left(g_{m p}\right)_{I} \cos \alpha^{*} s\right]\right.$

$$
\left.\cdot\left(\alpha^{*^{2}}+\gamma^{*^{2}}(L / b)^{2}\right)^{1 / 2} \cdot J_{1}\left[\left(\alpha^{*^{2}}+\gamma^{*^{2}}(L / b)^{2}\right)^{1 / 2} s / M\right] d \alpha^{*}\right\} d \gamma^{*}
$$


and

$$
\begin{aligned}
I_{m n p q}=-\frac{1}{\pi^{2}} \frac{1}{M^{2}} \int_{0}^{\infty}\left(g_{n q}\right)_{R}\{ & \int_{0}^{\infty}\left[\left(g_{m p}\right)_{R} \cos \alpha^{*} s+\left(g_{m p}\right)_{I} \sin \alpha^{*} s\right] \\
& \left.\cdot\left(\alpha^{*^{2}}+\gamma^{*^{2}}(L / b)^{2}\right)^{1 / 2} \cdot J_{1}\left[\left(\alpha^{*^{2}}+\gamma^{*^{2}}(L / b)^{2}\right)^{1 / 2} s / M\right] d \alpha^{*}\right\} d \gamma^{*} .
\end{aligned}
$$

Note that $H_{m n p q}$ and $I_{m n p q}$ are "admittance functions" which may be evaluated once and for all. Also $H_{m n p q}(s)=I_{m n p a}(s)=0$ for $s>M /(M-1)$ when $M>1$.

III. Discussion. Perhaps a brief word is in order with regard to the motivation for the manner in which the several integrations were carried out. The object, of course, is to minimize the amount of numerical work required. With the approach used it is only necessary to evaluate numerically the double integrals of $H_{m n p q}$ and $I_{\text {mnpq }}$. With any other order of inversion and/or integration at least two additional numerical integrations will be required which would make the determination of quantitative results impractical with presently available computational facilities.

Here we also quote an important special case of the general result, Eq. (10).

For simple harmonic motion, i.e.,

$$
a_{m n}(s)=\hat{a}_{m n} e^{i k s}
$$

and

$$
Q_{m n p q}(s)=\hat{Q}_{m n p q} e^{i k s}
$$

one may determine that

$$
\hat{Q}_{m n p q}=-\frac{1}{4 \pi^{2}} \hat{a}_{m n} \int_{-\infty}^{\infty} \int_{-\infty}^{\infty} \frac{\left(k+\alpha^{*}\right)^{2}}{\hat{\mu}} G_{m n p Q} d \alpha^{*} d \gamma^{*}
$$

where

$$
\hat{\mu} \equiv\left[-\gamma^{*^{2}}(L / b)^{2}-\alpha^{*^{2}}+M^{2}\left(\alpha^{*}+k\right)^{2}\right]^{1 / 2}
$$

Other methods [1] are available which determine $\hat{Q}_{m n p q}$; however, they are far more time consuming than the numerical evaluation of the double integral of Eq. (11). Also note that the present method is applicable to both subsonic and supersonic flow. On the other hand, these methods will treat the more difficult mixed-boundary value problem (for simple harmonic motion), i.e.,

$$
\left.\begin{array}{l}
\partial \phi / \partial z \text { known on plate } \\
p \text { continuous off plate }
\end{array}\right\} \text { on } z=0
$$

Finally we mention that for the related problem of arbitrary motion of a cylindrical shell (simple harmonic motion has been treated in References 3 and 4) the simplest procedure is to invert from the time domain numerically via a Fourier Transform. As demonstrated in the cited references [3, 4] the inversion to the spatial domain for the cylinder is relatively easy (easier than for the plate!), particularly for supersonic flow. (This is a result of being able to synthesize a solution for the cylinder by a Fourier series in the circumferential direction.) Thus a numerical inversion from the time domain for the cylindrical shell problem is a feasible and relatively economical calculation. 
IV. Numerical results. Representative numerical results will be presented for a family of plate deformations,

$$
\psi_{m}(\xi) X_{n}(\eta)=\sin m \pi \xi \sin n \pi \eta .
$$

The time dependence will be taken to be a unit step function,

$$
\begin{aligned}
a_{m n}(s)=1 & \text { for } s>0, \\
=0 & \text { for } \quad s<0 .
\end{aligned}
$$

In Figs. 2 and 3 are presented results for $Q_{1111}$ and $Q_{1211}$ for $L / b=1$ and several Mach

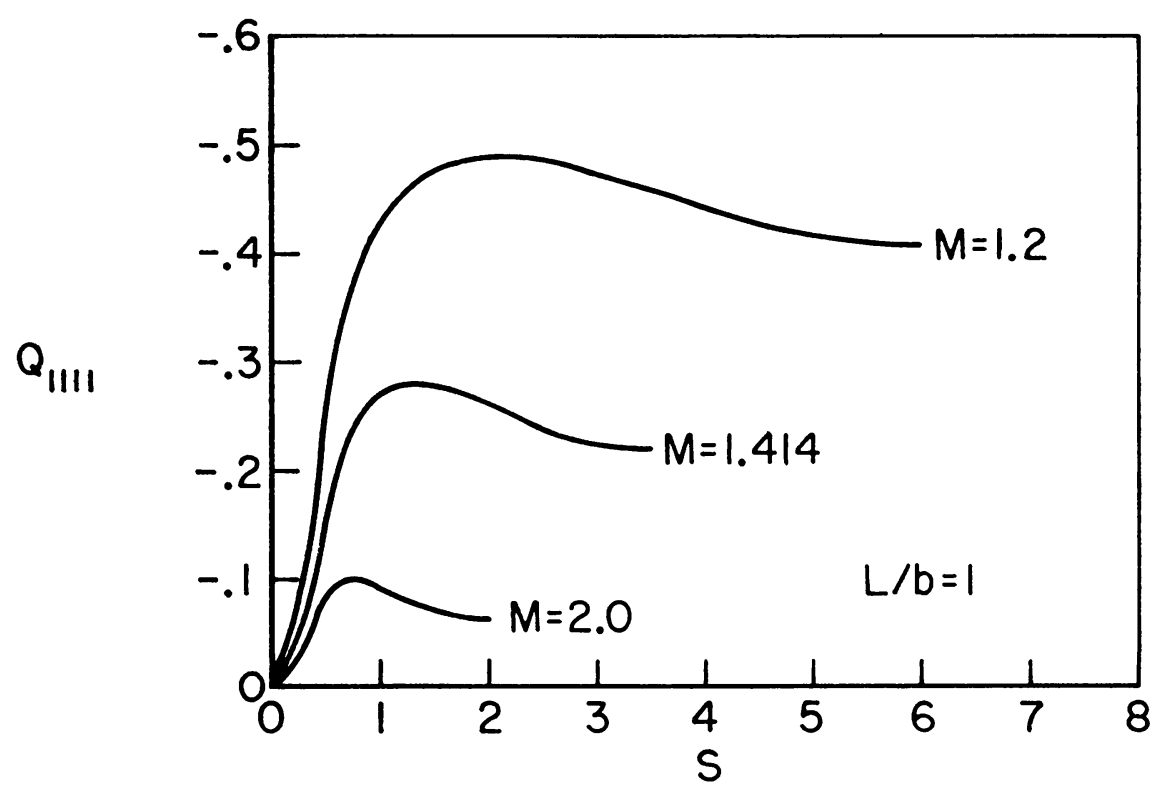

FIg. 2. Generalized Force vs. Time

numbers. In Figs. 4 and 5 results are shown for various $L / b$ for $M=1.414$. These data have a number of interesting features among which are:

(i) The $t=0^{+}$results are those given by "piston theory" [5] and are independent of $L / b$.

(ii) For supersonic flow the fluid has a finite memery of duration $M /(M-1)$ and thus for $s>M /(M-1)$ the values of $Q_{m n p q}$ do not change.

(iii) $Q_{1111}$ is much more sensitive to $L / b$ than $Q_{1211}$.

(iv) For $(L / b) /\left(M^{2}-1\right)^{1 / 2}<1 Q_{1211}$ has essentially its two-dimensional $(L / b=0)$ value.

(v) As $L / b$ and/or $M$ increases the generalized forces reach their asymptotic (large $s$ ) values more quickly.

From the behavior of $Q_{1111}$ and $Q_{1211}$ qualitative conclusions may be drawn about the stability of the system. However, such a discussion will be omitted here. 
The present method has also been used to independently calculate simple harmonic motion forces for a plate and also the admittance functions for a two-dimensional, supersonic airfoil* for which the present method is applicable. These results (not shown) agree with the known values. (see, for example, Reference 5.)

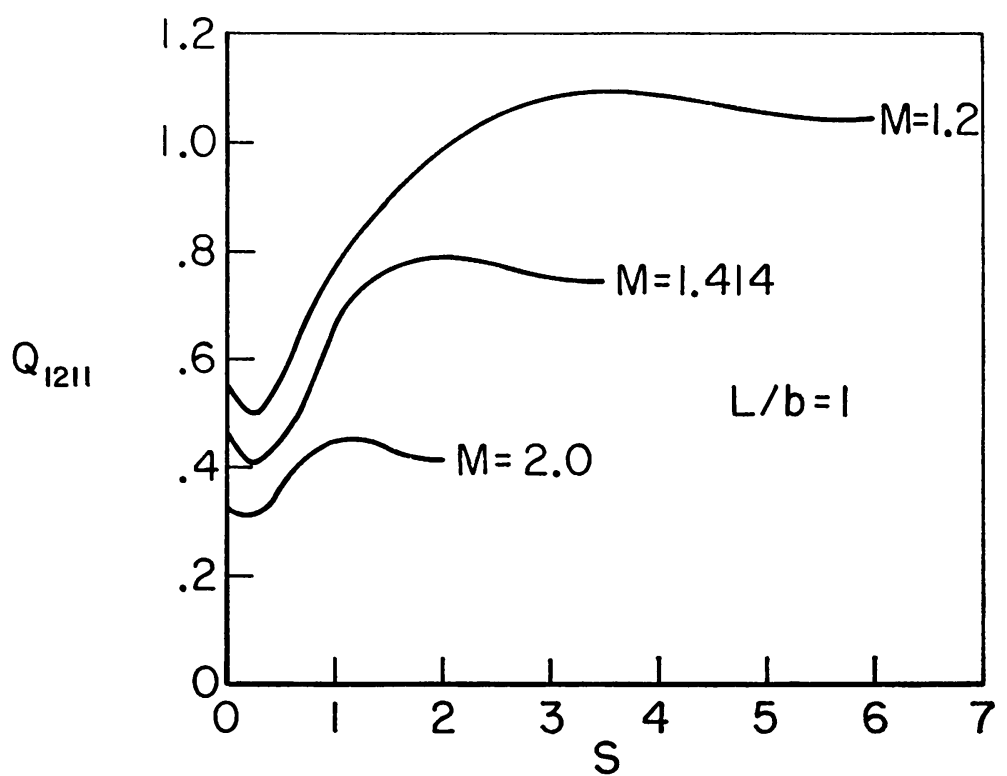

Fig. 3. Generalized Force vs. Time

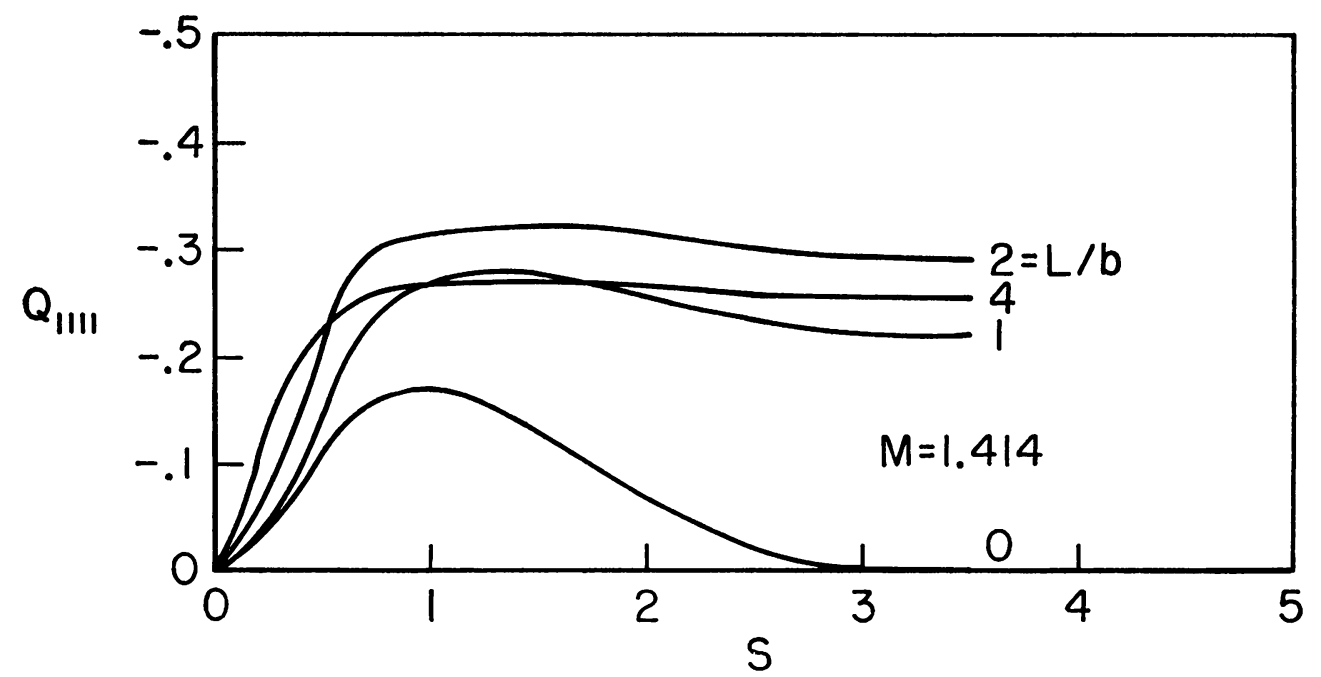

FIg. 4. Generalized Force vs. Time

*One must account for the fact that $\psi_{m}(0) \neq 0$, etc., in determining $f^{*}$. 


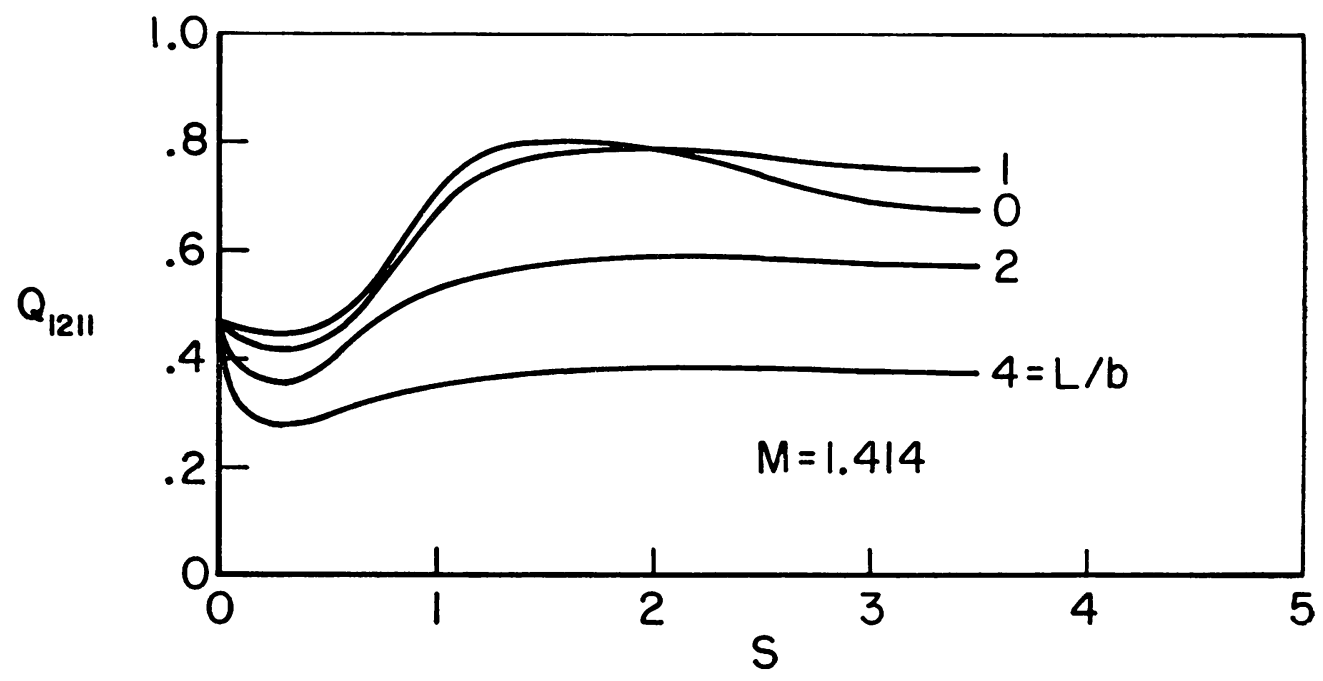

FIG. 5. Generalized Force vs. Time

Acknowledgment. This work was supported by NASA, Grant N6R31-001-059.

\section{REFERENCES}

1. H. J. Cunningham, Flutter analysis of flat rectangular panels based on three-dimensional supersonic potential flow, AIAA Journal (8) 1, 1795-1801 (August 1963)

2. Bateman Manuscript Project, T'ables of integral transforms, Vol. 1, McGraw-Hill, New York, 1954

3. E. H. Dowell and S. E. Widnall, Generalized aerodynamic forces on an oscillating cylindrical shell, Q. Appl. Math. 24, 1-17 (1966)

4. E. H. Dowell and S. E. Widnall, Generalized aerodynamic forces on an oscillating cylindrical shell: Subsonic and supersonic flow, AIAA Journal (4) 4, 607-610 (April 1966)

5. R. L. Bisplinghoff, H. Ashley and R. L. Halfman, Aeroelasticity, Addison-Wesley, Cambridge, Mass., 1955 